\title{
Identification of HMW-GS for development system evaluation bread-making qualities in early breeding generations in spring-sown triticale
}

Gruzdev I.V. ${ }^{1,2 *}$, Kolenkov M.A. ${ }^{1}$, Ermolenko O.I. ${ }^{3}$, Soloviev A.A. ${ }^{1,3}$

${ }^{1}$ All-Russia Research Institute of Agricultural Biotechnology, Moscow, Russia

${ }^{2}$ Russian Timiryazev State Agrarian University, Moscow, Russia

${ }^{3}$ N.V. Tsitsin Main Botanical Garden RAS, Moscow, Russia

*email: gruzdev82mtz@mail.ru

Triticale $(\times$ Triticosecale Wittm.) has a high yield potential, as well as increased resistance to biotic and abiotic environmental factors. The technological qualities of triticale flour are largely determined by the characteristics of the storage proteins of the grain. High molecular weight glutenin submints (HMW-GS) are of particular importance among storage proteins. In wheat, the contribution of high molecular weight glutenins to bread-making quality has been studied in the most detailed way and can even be expressed in numerical points. The bread-making qualities of wheat are largely determined by the HMW-GS encoded by the Glu-Dl locus, which in hexaploid triticale is replaced by the rye Glu-Rl genome locus. The polymorphism of the Glu-RI locus in hexaploid triticale has been fairly well studied; however, the effect of the alleles of this locus on the bread-making and technological properties of triticale flour has been poorly studied. Solving this issue would allow to optimize the selection of triticale genotypes with good and excellent bread-making qualities in the early generations. The identification of HMW-GS was performed by SDS-PAGE according to Singh et al. (1991), in 36 varieties and lines of spring hexaploid triticale for which a number of indicators were previously determined that indirectly affect the baking quality. The most widespread allelic state of the Glu-Rl locus was the combination of subunits $6^{\mathrm{r}}+13^{\mathrm{r}}$; the combinations $1^{\mathrm{r}}+4^{\mathrm{r}}$ and $2^{\mathrm{r}}+6,5^{\mathrm{r}}$ are slightly less common. An average negative relationship was found between the allelic composition of the Glu-Rl locus and the indicators of grain vitreousness, gluten quality, grain unit, and 1000 grain weight. Triticale can become a valuable raw material for the bakery industry, but for this it is necessary to eliminate the objective negative influence of the rye genome.

Acknowledgments: This research was funded by the Ministry of Education and Science of Russian Federation (Goszadanie No. 0431-2019-0005). 